\title{
Pathology of paediatric bone tumours
}

Adrienne M Flanagan MB FRCPath PhD FMedSci is Professor of Musculoskeletal Pathology at UCL Cancer Institute and Consultant pathologist at the Royal National Orthopaedic Hospital

Dr Luis Perez-Casanova, MB, Affiliated to the RCPath, is Pathology Fellow at the Royal National Orthopaedic Hospital

Postal address for both authors.

Department of Histopathology Institute of Orthopaedics, Royal National Orthopaedics Hospital

Brockley Hill, Stanmore

Middlesex

HA7 4LP

United Kingdom

Conflict of interests: none declared.

\begin{abstract}
Primary bone tumours account for less than $0.2 \%$ of all neoplasms but malignant bone tumours represent the $3^{\text {rd }}$ most common cause of cancer deaths in children and adolescents. The rarity of bone tumours in itself is a diagnostic challenge but is compounded by the number of tumour subtypes on top of which the imaging and histological features of degenerative and reactive processes, and benign bone tumours can simulate bone sarcomas. Furthermore, even in children bone lesions may represent metastatic disease. Hence the assessment of a bone tumour in a child or adolescent should be performed in a specialist referral bone tumour centre which has access to a multidisciplinary team and molecular diagnostic tests: the latter provides greater diagnostic accuracy. It is now appreciated that germline alterations occur more commonly than previously recognised in children and young adults presenting with osteosarcoma and Ewing sarcoma. Awareness of this is important as genetic counselling and screening may be appropriate. In this article epidemiology, radiology, pathology, genetics, treatment and prognosis of most commonly encountered bone tumours among the paediatric population are reviewed.
\end{abstract}

Keywords: Bone tumour, osteosarcoma, chondrosarcoma, chordoma, Ewing sarcoma, mutation, genetics, children, paediatric, sarcoma, mosaic disorder 


\section{INTRODUCTION}

Primary bone tumours represent between $4.6 \%$ and $7.6 \%$ of all cancers in patients under 20 years of age $[1,2]$. Most paediatric primary bone tumours are benign, however malignant bone tumours account for significant morbidity and mortality in this population. A recent publication reports that the prevalence of germline mutations in sarcoma in patient presenting under 20 years of age come to $8 \%$ but occurs in closer to $20 \%$ and $10 \%$ of patients in this age group with osteosarcoma and Ewing sarcoma respectively [2]. Pathological assessment of a bone tumour in a child or adolescent should be performed by histopathologists in a specialist bone tumour referral centre .

In this review we will focus on primary bone tumours that are most frequently encountered in the paediatric population. A classification of these tumours is shown in Table 1.

\section{BENIGN BONE TUMOURS}

\section{BONE-FORMING TUMOURS}

\section{Osteoma and enostosis}

Definition, Epidemiology and Incidence

Osteoma is a benign tumour occurring on the bone surface, and is referred to as enostosis (bone island) when in the medullary cavity. The sporadic incidence is $0.4 \%$ although in patients with Gardner syndrome the incidence is approximately $24 \%$. There is a male predominance (male to female ratio 2:1). Typically arising on the surface of craniofacial bones (jaws, frontal, nasal sinuses) it may block nasal ducts causing headache pain, or exophthalmos [3].

\section{Pathology}

Histology; a thick mass of mature compact bone with decreased marrow space.

Differential Diagnosis

Periosteal osteoblastoma, sessile osteochondroma and parosteal osteosarcoma.

\section{Genetics}

Multiple osteomas are characteristic of Gardner syndrome: this autosomal dominant (AD) disorder should also be considered when an osteoma presents in the long bones. Gardner syndrome is a variant of familial adenomatous polyposis.

\section{Prognosis}

No reports of malignant transformation. Most osteomas require no treatment and do not recur if excised.

\section{Osteoid Osteoma}

Definition, Epidemiology and Incidence 
A benign intracortical bone-forming tumour with a male predilection (3:1) accounting for 10$15 \%$ of all primary osseous tumours. Characteristically it presents with night pain, effectively relieved with non-steroidal anti-inflammatory drugs. The signs vary depending on the site: scoliosis in the spine, limping and joint swelling resembling arthritis when the end of long bones, and loss of function in the small bones of the hands.

\section{Radiology and Pathology}

Radiology shows a small lucent nidus, less than $2 \mathrm{~cm}$, with a peripheral area of sclerosis.

Figure 01 for microscopic features. The differential diagnoses include stress fracture, a bone island and osteoblastoma.

\section{Treatment and Prognosis}

No risk of malignant transformation. Surgical removal, or now more commonly minimally invasive procedures, to ablate the nidus is curative, and results in immediate pain relief.

\section{Osteoblastoma}

\section{Definition, Epidemiology and Incidence}

A benign bone-forming intramedullary tumour although rarely periosteal-based accounting for $1 \%$ of all primary osseous tumours. There is a male predilection (2.5:1) and presents most commonly in a child or young adult and less commonly later in life. Pain is localized but in contrast to osteoid osteoma it is not characteristically nocturnal nor does it respond consistently to non-steroidal anti-inflammatory agents. Most commonly seen in the axial skeleton, $40 \%$ arise in the posterior elements of the spine and sacrum but they can also occur in the metaphysis of long bones most commonly femur and tibia.

\section{Radiology and Pathology}

Radiographically: an irregular, radiolucent lesion with variable patchy intra-tumoural mineralization. The oedema characteristic on imaging can also be seen associated with Ewing sarcoma, infection. Tumour size varies from 2 to greater than $10 \mathrm{~cm}$. Histologically osteoblastomas are not dissimilar to osteoid osteoma but can generally be distinguished by their size $(>2 \mathrm{~cm})$ and their medullary site. Rarely the tumour cells are very atypical when referred to as 'epithelioid', 'bizarre' and 'aggressive' osteoblastomas but the behaviour of such lesions is not different from those with more conventional histology [3].

\section{Differential diagnosis}

Osteoid osteoma, high-grade osteosarcoma, haemangioma.

\section{Treatment and Prognosis}

No risk of malignant transformation. Complete excision is curative and surgical removal by curettage is the treatment of choice for lesions of the long bone but en bloc resection may be required for lesions of the vertebra. 


\section{CARTILAGE-FORMING TUMOURS}

1. Osteochondroma

Definition, Epidemiology and Incidence

Osteochondroma is a benign cartilage-capped bony exostosis with a male predominance (2:1). The marrow in the underlying bone continues directly into the stalk of the exostosis. It is one of the most common benign bone tumours, but the true incidence is unknown as many patients are asymptomatic. It presents either as a lump or with pain and can arise in any bone that undergoes endochondral ossification, but frequently in the femur, humerus, tibia and fibula [3].

Radiology and Pathology

Typically presents as a solitary metaphyseal exophytic tumour either sessile or pedunculated. Multiple osteochondromas occur on a background of a germline disorder.

An osteochondroma is composed of a hyaline cartilage cap, covered by perichondrium, and connected to the underlying bone marrow. The cartilage cap continues to grow while the growth plate is open and can reach up to $5 \mathrm{~cm}$. As the skeleton reaches maturity the chondrocytes die and the cartilage cap calcifies resulting in only a thin rim of a persistent cartilage cap $(<1 \mathrm{~cm})$. Continued growth of the tumour in the mature skeleton and an uncalcified cartilage cap in the mature skeleton of $>3 \mathrm{~cm}$ are worrying feature and suggests transformation to a chondrosarcoma.

Genetics and diagnostic tests

Multiple osteochondromas can be seen in hereditary forms such as Multiple Osteochondromas (AD disorder with EXT1 and EXT2 germline alterations), Langer-Giedion syndrome and DEFECT-11 syndrome. These hereditary forms can result in bony deformity, restricted joint motion and short stature. Genetic counselling is recommended as is a followup plan for children transferring to an adult clinical service.

\section{Differential Diagnosis}

Bizarre parosteal osteochondromatous proliferation (BPOP, AKA Nora's lesion), subungual exostosis, periosteal chondoma, parosteal osteosarcoma.

BPOP is a small (usually $<2 \mathrm{~cm}$ ) exostosis presenting most commonly on the finger. Subungual exostosis occurs most frequently under the great toe. Both show similar histological features, including hypercellular cartilage merging with underlying reactive bone. Local recurrence is common. Malignant transformation has not been reported [3].

Treatment and Prognosis

A minority of osteochondromas progress to peripheral chondrosarcoma ( $1 \%$ for solitary and $5 \%$ for multiple osteochondromas) [3], but the risk of sarcomatous progression in skeletally 
immature patients is much lower than in adults, although rare cases have been reported. The treatment of choice is surgical removal.

\section{Enchondroma/Enchondromatosis}

Definition, Epidemiology and Incidence

Enchondroma is an intramedullary benign hyaline cartilage tumour representing $24 \%$ of all bone tumours in this age group. The real incidence is higher as many tumours are found incidentally [3]. It affects both sexes equally and can present at any age. They most frequently involve the small tubular bones of the hands and feet, but can be seen in any bone. They can present with local pain, swelling if on the hands and feet, and pathologic fracture.

Enchondromas are usually solitary, but multiple enchondromas can present as part of multiple syndromes, the most common being Ollier disease and Maffucci syndrome (vide infra) [4,5]. Other forms of multiple enchondromas include

Ollier disease and Maffucci syndrome are overlapping mosaic disorders characterised by the presence of multiple enchondromas virtually always involving the small bones of the hands and or feet but other bones are also affected [5]. The number of tumours range from a small number to hundreds. Maffucci syndrome is characterised by spindle cell haemangiomas in addition to enchondromas. These benign cartilage tumours result in bone deformities, and short stature. Individuals are also at risk of developing other tumours, mainly glial brain tumours [6].

\section{Pathology}

Enchondromas are composed of cellular lobules of abundant mature hyaline cartilage containing chondrocytes with no significant cytological atypia. Mitotic figures are not seen.

\section{Diagnostic tests}

Isocitrate dehydrogenase type 1 or type 2 (IDH1/IDH2) somatic point mutation is detected in $60 \%$ of solitary cases and in $>90 \%$ of patients with Ollier disease, and Maffucci Syndrome.

If the characteristic IDH1/IDH2 mutation is not identified in a patient with Ollier disease another more rare form of Multiple Enchondromas should be sought, such as metachondromatosis and spondyloenchondrodysplasia (germline genetic alterations PTPN11 and $A C P 5$ respectively); such patients benefit from genetic screening/ counselling.

\section{Differential Diagnosis}

Fibrous dysplasia with cartilaginous differentiation, chondrosarcoma.

\section{Treatment and Prognosis}

Malignant transformation in solitary enchondromas is rare. However there is significant risk, as high as $15 \%$ to $30 \%$ in Ollier disease and Maffucci syndrome, of developing malignant 
transformation in one of their multiple lesions. The risk of this occurring in childhood is low [6].

The treatment of enchondromas depends on the symptoms and size of the lesion. Incidental solitary enchondromas of the long or flat bones can simply be monitored radiologically if asymptomatic. Curettage of enchondromas of the small bones of the hands and feet should be curative. Recurrence is extremely uncommon. Children with multiple enchondromas should be monitored closely and consideration should be given as to how best support these patients when transferred to an adult clinical service.

\section{Chondroblastoma}

\section{Definition, Epidemiology and Incidence}

Chondroblastoma is a benign intramedullary non-conventional cartilaginous tumour. It account for $<1 \%$ of all bone tumours [3,7] and typically presents in the epiphysis of long bones of skeletally immature individuals, more commonly in males. It presents with localized pain.

\section{Radiology and Pathology}

Radiographically: well-circumscribed lytic lesions, with a sclerotic margin, and central radiodensities. The microscopic - Figure 02.

Diagnostic tests

Somatic driver mutations H3F3A and H3F3B (p.K36M) genes are found in $>90 \%$ of chondroblastomas, for which a diagnostic antibody available $[11,16]$.

Differential Diagnosis

Aneurysmal bone cyst, giant cell tumour of bone, chondroblastoma-like osteosarcoma [7].

\section{Treatment and Prognosis}

Very rare cases of malignant transformation and metastasis have been reported [3].

Curettage with or without bone grafting: local recurrence rates up to $18 \%$.

\section{Chondromyxoid fibroma (CMF)}

Definition, Epidemiology and Incidence

A benign intramedullary non-conventional cartilaginous tumour accounting for less than $1 \%$ of all bone tumours. It presents most often in the first 3 decades of life with a slight male predominance (1.5:1). Patients usually present with a long-term history of pain.

It can arise in any bone, and is most often located in the metaphysis of long bones.

Radiology and Pathology 
Histology: lobules of spindle to stellate cells set in a fibromyxoid matrix. Large, bizarre hyperchromatic nuclei are seen in $20 \%$ to $30 \%$ of cases which can make the diagnosis challenging [3]. The radiological features are not specific.

\section{Genetics and diagnostic tests}

Most cases are characterised by a somatic GRM1 gene rearrangement.

\section{Differential diagnosis}

Other conventional and non-conventional benign and malignant cartilaginous tumours. However, chondrosarcoma occurs rarely in children.

\section{Treatment and Prognosis}

CMF have an excellent prognosis: no reports of malignant transformation. Treated by curettage and bone grafting although there is a local recurrence of up to $15 \%$ [3].

\section{FIBROUS AND OSTEOFIBROUS BONE LESIONS}

\section{Non-Ossifying Fibroma (NOF)}

Definition, Epidemiology and Incidence

NOF is an intracortical benign fibroblastic lesion, also referred to as a "fibrous cortical defect". It is most commonly located in the metaphysis of long bones of skeletally immature patients, occurring twice more often in males. Usually asymptomatic and often discovered incidentally, although studies have reported incidences from 30 to $40 \%$ in children and adolescents [3]. Pathologic fracture is the main presenting symptom in larger tumours.

\section{Pathology}

Sheets of bland spindle cells with a storiform growth pattern, scattered osteoclast-like cells and collections of foamy histiocytes.

Genetics and diagnostic tests

Multifocal NOF may occur in Neurofibromatosis type 1, and Jaffe-Campanacci syndrome.

Differential Diagnosis

Fibrous dysplasia, osteofibrous dysplasia.

Treatment and Prognosis

Malignant transformation has not been reported. If symptomatic or at risk of fracture $(>50 \%$ of bone width), curettage is the treatment of choice. Children with McCune Albright syndrome should be monitored closely and consideration as to how best to support these patients when transferred to an adult clinical service. 


\section{Fibrous Dysplasia (FD)}

Definition, Epidemiology and Incidence

A benign fibro-osseous lesion mosaic disorder usually presenting in patients younger than 30 years and affects both genders equally. The lesions may occur in single (monostotic) or multiple bones (polyostotic). McCune-Albright syndrome is characterised by FD, endocrine abnormalities and café-au-lait spots. Mazabraud syndrome refers to patients with fibrous dysplasia and intramuscular myxoma $[3,9]$.

Mostly asymptomatic however, FD can present with pain, swelling, bone deformities or pathologic fractures. FD can occur in any bone, with a predilection for the craniofacial and long bones.

\section{Radiology and Pathology}

On plain films FD characteristically shows a ground-glass appearance with bone expansion.

Figure 03 for microscopic features.

Diagnostic tests

Somatic point mutations in the GNAS (R201C, R201H, Q227L) in >90\%.

Differential Diagnoses

Osteofibrous dysplasia, low-grade central osteosarcoma.

Treatment and Prognosis

Malignant transformation occurs in less than $1 \%$ of cases.

Treatment is often not required in asymptomatic solitary lesions of FD, and the prognosis is excellent. Treatment is determined by symptoms and bone deformity. Bisphosphonates can be useful for controlling pain. Referral to a specialist physician is recommended for pain control. Consideration should be given as to how best to support these patients when transferred to an adult clinical service.

\section{Osteofibrous Dysplasia (OFD)}

Definition, Epidemiology and Incidence

A rare fibro-osseous lesion of bone representing $0.2 \%$ of primary bone tumours involving the tibia, and sometimes fibula, presenting in children and infants, with no clear sex predilection $[3,8]$.. It usually presents as a painless area of swelling on the tibial anterior surface.

Radiology and Pathology

Characteristic radiological image of an anterior cortical lucent lesion of the tibial diaphysis. 
The microscopic and immunohistochemical features - Figure 04.

Genetics and diagnostic tests

Germline MET point mutations have been identified in familial and bilateral OFD.

Differential Diagnosis

Fibrous dysplasia, non-ossifying fibroma.

\section{Treatment and Prognosis}

There is no consensus how to best treat OFD. Progression into adamantinoma, a malignant disease, has been reported is some patients. [3] Follow-up is recommended as a minimum.

\section{OSTEOCLAST-RICH TUMOURS}

\section{Primary Aneurysmal Bone Cyst (ABC)}

Definition, Epidemiology and Incidence

A locally destructive, benign intramedullary expansile, cystic neoplasm and composed of multiple septated blood-filled cavities. Almost $80 \%$ of the lesions are found in patients younger than 20 years old: both genders are affected equally $[3,10]$. $A B C$ typically causes localized pain, swelling and may present as a pathologic fracture. Lesions in the spine may be associated with neurologic symptoms. Lesions crossing the growth plate may cause growth arrest. $A B C$ can arise in any bone, but the most common location is the region around the knee.

\section{Pathology}

Haemorrhagic, multiple blood-filled cavities separated by fibrous septa composed of bland stromal spindle cells and scattered osteoclast-type giant cells. Osteoid and 'blue' bony matrix may be prominent. In some cases the tumour is predominantly solid with only a minor cystic component.[3]

Diagnostic tests

Cytogenetic rearrangements involving the UPS6 gen e are found in approximately $70 \%$ of primary $A B C$ [3]. A diagnostic genetic is test available.

Differential Diagnosis

$\mathrm{GCT}$, telangiectatic osteosarcoma, secondary $\mathrm{ABC}$ (vide infra) $[7,10,11]$.

\section{Treatment and Prognosis}

Curettage with bone grafting provides an excellent outcome in most cases. 


\section{Secondary Aneurysmal Bone Cyst}

This term applies when aneurysmal cyst changes occur in association and secondary to other bone tumours most of which are benign. Unexpected expansion of neoplasms that are most frequently seen with secondary $A B C$ such as chondroblastoma, giant cell tumour of bone, $\mathrm{CMF}, \mathrm{FD}$ and haemangioma is often explained by secondary aneurysmal bone cyst formation. CT-guided biopsy provides the best chance of sampling the relevant area of the tumour.

Treatment is tailored to the primary disease. UPS6 alterations are not associated with secondary $A B C$.

\section{Giant Cell Tumour of Bone (GCT)}

A benign, locally aggressive intramedullary neoplasm characterised by abundant osteoclastlike giant cells. GCT only occur in an immature skeleton in exceptionally rare cases so it will not be discussed here in detail. Serum chemistry of calcium and phosphate should be undertaken to exclude a metabolic disorder particularly hyperparathyroidism. More than $90 \%$ of GCT harbour a somatic driver mutations the histone variant $\mathrm{H} 3.3$ gene, $>90 \%$ involving p.G34W and occasionally p.G34L substitution. These mutations are valuable for diagnostic purposes [11].

\section{MALIGNANT BONE-FORMING TUMOURS - OSTEOSARCOMA}

\section{Definition, Epidemiology and Incidence}

It is the most common primary malignant bone-forming tumour occurring in children and adults, more commonly in males[1]. Approximately $70 \%$ present before the age of 40 between the ages of 10 and 14 but rarely before the age of 4 . The incidence is $4.4 /$ million between the ages of $4-24$.

Patients present with pain, particularly at night, and less commonly fracture. The pain can be dismissed as 'growing pains' resulting in a delayed diagnosis.

\section{Pathogenesis and Aetiology}

In young people osteosarcoma occurs without a known cause but a recent publication reports that close to $20 \%$ of patients under the age of 20 harbour a germline alteration giving rise to clinical familial syndromes particularly TP53 alterations in Li-Fraumeni, RB1 alterations in hereditary retinoblastoma syndrome, RECQL4 in Rothmund-Thomson syndrome, and TNFSRA11A in familial Paget's disease $[3,12]$. Consideration should be given to genetic counselling, and screening in children with osteosarcoma. Most (90\%) osteosarcomas arise in the bone (central osteosarcoma) and more rarely on the bone (surface osteosarcoma 5-10\%) although the latter rarely occur in children. Most osteosarcomas in children are high-grade and like other sarcomas metastasise to the lungs.

\section{Pathology and Radiology}

High-grade central osteosarcoma comprises a variety of histological features but in general are characterised by osteoid production by highly atypical tumour cells; mitotic activity is 
generally high and there is often extensive necrosis. There are a number of histological subtypes the most common being the osteoblastic subtype then chondroblastic and less commonly fibroblastic/ pleomorphic, telangiectatic, giant cell-rich and small cell type. However, many osteosarcomas show a mixed histological picture and these subtypes do not inform on prognosis or predict response to chemotherapy (Figs 5, 6). High-grade surface osteosarcoma is exceptionally rare in any age range.

\section{Differential Diagnosis}

Osteosarcoma is a histological mimic of many tumours, both benign and malignant, and also non-tumour entities. The list of differential diagnoses is large but the following are the most frequently experienced challenges. Fracture callus has many histological similarities to a chondroblastic osteosarcoma, the osteolytic processes of infection and non-infectious osteomyelitis may mimic osteosarcoma, and the metabolic disorder of hyperparathyroidism can share histological and radiological features of an osteosarcoma.

Distinguishing a chondroblastic osteosarcoma from a chondrosarcoma (rarely occurring in children) is also important as the former is treated with neoadjuvant chemotherapy whereas there is no evidence that the latter responds to such treatment.

Distinguishing between aneurysmal bone cyst and a telangiectatic osteosarcoma, and fibrous dysplasia and a low-grade osteosarcoma can be extremely difficult. See section on metastatic disease

\section{Treatment and Prognosis}

The standard of care treatment for high-grade osteosarcoma is neoadjuvant chemotherapy (methotrexate, doxorubicin, and cisplatin) followed by surgery. With recent advances limbsparing surgery is more common than amputation. The best predictor of survival is the presence of at least $90 \%$ tumour necrosis assessed on histological post-surgery specimens. Five years survival of patients whose tumours show a 'good response' and who present without metastatic disease is $\sim 60 \%$, whereas a 'poor-response' is associated with survival rates of less than $15 \%$. Patients with metastatic or recurrent disease have survival rates of $<$ $20 \%[1]$.

Low-grade osteosarcoma is treated with wide en bloc wide excision but. Diligent sampling of the specimen is required, as is inspection of the margin of excision, as low-grade osteosarcoma may transform to a high grade disease [1].

\section{MALIGNANT CARTILAGE-FORMING TUMOUR}

Primary conventional chondrosarcoma rarely occurs in children so will not be discussed further.

Mesenchymal chondrosarcoma (MCS) occurs most commonly in the second and third decades, with no sex predilection [1]. It presents predominantly in the craniofacial bones, especially the jaw, and long bones of teenagers and young adults. The vertebrae, ribs, and 
pelvis are also common sites of involvement. Furthermore, up to $1 / 3^{\text {rd }}$ of cases occur in soft tissue [3].

\section{Pathology and Imaging}

Imaging: findings are not specific and demonstrate bone expansion, cortical destruction and soft tissue involvement all of which are can be associated with osteosarcoma and Ewing sarcoma.

Histology: a biphasic high-grade tumour composed of variably amounts of differentiated cartilaginous islands admixed with a 'small round blue cell' component [13].

\section{Diagnostic tests}

AEY1-NCOA2 fusion; IDH1 and IDH2 point mutations are absent.

\section{Differential Diagnosis}

'Small round blue cell tumours' such as Ewing family of tumours, small cell osteosarcoma, lymphomas, Langerhans cell histiocytoma (LCH), and metastatic disease (vide infra).

\section{Prognosis and Treatment}

Neoadjuvant chemotherapy and surgical excision is the primary treatment of choice. Metastases often appear late in the course of the disease (even 20 years after initial diagnosis [3]). The overall survival is poor at $54.6 \%$ and $27.3 \%$ at 5 and 10 years respectively.

\section{'SMALL ROUND BLUE CELL TUMOURS'}

\section{Ewing sarcoma (ES)}

Definition, Epidemiology and Incidence

Ewing sarcoma accounts for $6-8 \%$ of primary malignant bone tumours with an estimated $10 \%$ occurring on a background of a germline alteration [2,3]. Males are most commonly affected, and Ewing sarcoma of bone occurs mostly in children or adolescents aged less than 20 years. This is in contrast to soft tissue Ewing sarcoma which occurs more commonly in adults. It is the second most common bone sarcoma in the paediatric age group after osteosarcoma. Ewing sarcoma rarely occurs in the black African population. It generally presents with pain and occurs in a long bone, pelvis, or rib. It presents in soft tissue more commonly in adults [14].

\section{Pathology and Radiology}

Imaging reveals an ill-defined lytic or sclerotic diaphyseal mass with discontinuous periosteal reaction (sunburst or onion-skin pattern). Histology shows a monotonous population of 'small round blue tumour cells' [3].

Diagnostic Tests 
Immunohistochemistry and detection of characteristic fusion genes is required to provide a diagnosis. Membranous expression of CD99 and nuclear FLI1 are supportive of the diagnosis of Ewing sarcoma, although these markers are not specific.

More than $90 \%$ of Ewing sarcoma reveal a EWSR1-FLI1 translocation and 5\% show EWSR1$E R G$ translocation; $<1 \%$ have other less frequent EWSR1 translocations and $<1 \%$ harbour non-EWSR1 translocations (FUS-ERG; CIC-DUX4; BRD4-NUT; BCOR-CCNB3).

\section{Differential Diagnosis}

Small cell osteosarcoma and mesenchymal chondrosarcoma, lymphoma, leukaemia, and metastatic 'round cell' tumours.

\section{Prognosis and Treatment}

Patients who at presentation have localized, resectable disease and treated with multidrug chemotherapy and surgery have a 70\% 5-year survival. For patients with disseminated disease at diagnosis the 5 -year survival rate is approximately $30 \%$ [12].

\section{MISCELANEOUS}

\section{Chordoma}

\section{Definition, Epidemiology and Incidence}

Chordoma is a slow-growing malignant neoplasm showing notochordal differentiation, accounting for $3-4 \%$ of primary malignant bone tumour [12]. Childhood chordoma accounts for $<5 \%$ of all chordomas. It affects males more frequently $(2: 1)$. Although they may arise anywhere in the midline skeleton from the base of skull to the coccyx, they most frequently occur in the spheno-occipital region in children. Rarely chordoma can be present at birth. It almost always presents with pain, other clinical manifestations may vary depending on the site of the lesion, but due to their slow growth they tend to be silent until an advance stage.

\section{Pathology}

A gelatinous, multilobulated tumour composed of vacuolated (physaliphorous) cells arranged in nests or chords, embedded in a myxoid matrix. Dedifferentiation is a rare event and is represented by loss of the characteristic histological features and replaced with a high-grade spindle cell sarcoma occasionally with osteosarcomatous, or rhabdomyosarcomatous differentiation [15].

\section{Diagnostic Tests}

Brachyury and cytokeratin immunoreactive, unless in the dedifferentiated form; S100 variable expression. Loss of INI1 is rare, mostly seen in children.

\section{Differential Diagnoses}

Chondrosarcoma, CMF, metastatic adenocarcinoma, lipomatous tumours. 


\section{Treatment and prognosis}

Complete surgical excision with or without adjuvant proton beam therapy is the treatment of choice. There is a high risk of local recurrence. Metastases occur late. The median survival is 7 years.

\section{Langerhans Cell Histiocytosis (LCH)}

Definition, Epidemiology and Incidence

A disease of dendritic cells and of unknown aetiology. It represents $<1 \%$ of all primary bone lesions [3] and $80 \%$ occur in the skeleton resulting in osteolytic lesions in one or more bones. It most frequently affects the skull but it can also affect other organs. The most common presenting symptom is pain and swelling.

\section{Diagnostic Tests}

The Langerhans are immunoreactive with CD68, S100, CD1a and CD207. The BRAF V600E mutation is detected in $60 \%$ of patients [3].

\section{Differential Diagnoses}

Osteomyelitis, granulomatous inflammation, parasitic and fungal infections, lymphoma.

\section{Prognosis and Treatment}

The most important adverse prognostic factors are presentation at $<2$ years of age and disseminated disease. The overall prognosis is excellent for both monostotic and limited polyostotic disease but recurrence is not uncommon. Lesions may resolve spontaneously or if persistent they can be treated with curettage. Multifocal bone disease, severe pain, or restricted mobility may require systemic chemotherapy: this can include prednisone, vinblastine; indomethacin, bisphosphonates and BRAF inhibitors.

\section{METASTASES}

Most childhood tumours that metastasize to bone are 'small round blue cell tumours' the most common originating from neuroblastoma, medulloblastoma, rhabdomyosarcoma, amongst others. Acute lymphocytic leukaemia or other haematological diseases can also present with disease in the bone marrow. Metastatic disease in bone can induce a prominent osteoblastic reaction and mimic an osteosarcoma challenging the diagnostic skills of radiologists and pathologists [12].

\section{REFERENCES}


[1] Whelan J, McTiernan A, Cooper N, Wong Y, Francis M, Vernon S et al. Incidence and survival of malignant bone sarcomas in England 1979-2007. International Journal of Cancer. 2011;131(4):E508E517.

[2] Zhang J, Walsh M, Wu G, Edmonson M, Gruber T, Easton J et al. Germline mutations in Predisposition Genes in Pediatric Cancer. New England Journal of Medicine. 2015;373(24):23362346.

[3] Fletcher C. WHO classification of tumours of soft tissue and bone. Lyon: IARC Press; 2013.

[4] Pansuriya T, van Eijk R, d'Adamo P, van Ruler M, Kuijjer M, Oosting J et al. Somatic mosaic IDH1 and IDH2 mutations are associated with enchondroma and spindle cell hemangioma in Ollier disease and Maffucci syndrome. Nature Genetics. 2011;43(12):1256-1261.

[5] Amary M, Damato S, Halai D, Eskandarpour M, Berisha F, Bonar F et al. Ollier disease and Maffucci syndrome are caused by somatic mosaic mutations of IDH1 and IDH2. Nature Genetics. 2011;43(12):1262-1265.

[6] Verdegaal SHM, Bovee JVMG, Pansuriya TC, Grimer RJ, Ozger H, Jutte PC, et al. Incidence, Predictive Factors, and prognosis of chondrosarcoma in patients with Ollier Disease and Maffucci Syndrome: An international multicenter study of 161 patients. The Oncologist.

2011Jan;16(12):1771-9.

[7] Presneau N, Baumhoer D, Behjati S, Pillay N, Tarpey P, Campbell P et al. Diagnostic value ofH3F3A mutations in giant cell tumour of bone compared to osteoclast-rich mimics. The Journal of Pathology: Clinical Research. 2015;1(2):113-123.

[8] Gray M, Kannu P, Sharma S, Neyt C, Zhang D, Paria N et al. Mutations Preventing Regulated Exon Skipping in MET Cause Osteofibrous Dysplasia. The American Journal of Human Genetics. 2015;97(6):837-847.

[9] Idowu B, Al-Adnani M, O'Donnell P, Yu L, Odell E, Diss T et al. A sensitive mutation-specific screening technique for GNAS1 mutations in cases of fibrous dysplasia: the first report of a codon 227 mutation in bone. Histopathology. 2007;50(6):691-704.

[10] Amary M, Ye H, Berisha F, Tirabosco R, Presneau N, Flanagan A. Detection of USP6 gene rearrangement in nodular fasciitis: an important diagnostic tool. Virchows Archiv. 2013;463(1):9798.

[11] Behjati S, Tarpey PS, Presneau N, Scheipl S, Pillay N, Loo PV, et al. Distinct H3F3A and H3F3B driver mutations define chondroblastoma and giant cell tumor of bone. Nature Genetics. 2013;45(12):1479-82.

[12] Czerniak. Dorfman and Czerniak's Bone Tumors. Elsevier; 2016.

[13] Bishop MW, Somerville JM, Bahrami A, Kaste SC, Interiano RB, Wu J, et al. Mesenchymal Chondrosarcoma in Children and Young Adults: A Single Institution Retrospective Review. Sarcoma. 2015;2015:1-6. 
[14] Antonescu C. Round cell sarcomas beyond Ewing: emerging entities. Histopathology. 2013;64(1):26-37.

[15] Vujovic S, Henderson S, Presneau N, Odell E, Jacques T, Tirabosco R et al. Brachyury, a crucial regulator of notochordal development, is a novel biomarker for chordomas. The Journal of Pathology. 2006;209(2):157-165.

[16] 1. Amary M, Berisha F, Mozela R, Gibbons R, Guttridge A, O'Donnell P et al. The H3F3 K36M mutant antibody is a sensitive and specific marker for the diagnosis of chondroblastoma. Histopathology. 2016;69(1):121-127.

\section{CAPTIONS}

Fig 1. Osteoid osteoma characteristically has a central nidus with anastomosing trabeculae of osteoid $(*)$ and woven bone with prominent osteoblastic rimming (arrow head), set in a loose vascular fibrous background with occasional osteoclasts (arrow).

Fig 2. Chondroblastoma is composed of round to oval chondroblastic cells with uniform, grooved nuclei (arrow) embedded in a chondromyxoid matrix, which shows the characteristic pericellular "chicken wire" calcification $(*)$.

Fig 3. Fibrous dysplasia. Bland spindle cells in which irregularly curved immature woven bone ("Chinese letters") lacking a rim of osteoblasts are embedded. The bone on the left of the picture is reactive bone surrounding the lesion.

Fig 4. Osteofibrous dysplasia is characteristically composed of curved woven bone trabeculae with conspicuous osteoblastic rimming (arrow) set in a bland fibrous stroma. B.A immunohistochemistry section showing scattered small non-clustered of epithelial cytokerkatin (MNF116) (brown stain) cells. If epithelial clustered cells or nests are clearly visible on H\&E-stained sections, or after immunostaining, the tumour should be designated as "OFD-like adamantinoma",

Fig 5. Osteoblastic osteosarcoma is composed of 'packed' severely atypical polygonal cells with lacelike osteoid deposition $(*)$. Numerous mitoses are seen (arrow head).

Fig 6. Telangiectatic osteosarcoma is composed of blood-filled cystic spaces separated by cellular areas where the cells show severe nuclear pleomorphism and scattered multinucleated tumour giant cells (arrow head). Osteoid matrix which is often sparse in this subtype of osteosarcoma is not present in this photomicrograph. 


\begin{tabular}{|c|c|c|}
\hline Main groups & Benign & Malignant \\
\hline \multirow{2}{*}{ Bone-forming tumours } & $\begin{array}{l}\text { Osteoma } \\
\text { Osteoid osteoma } \\
\text { Osteoblastoma }\end{array}$ & $\begin{array}{l}\text { CENTRAL } \\
\text { High grade central } \\
\text { - Osteoblastic } \\
\text { - Chondroblastic } \\
\text { - } \quad \text { Fibroblastic / } \\
\text { Pleomorphic } \\
\text { - Telangiectatic } \\
\text { - Giant-cell rich } \\
\text { - Small cell } \\
\text { Low grade central }\end{array}$ \\
\hline & & $\begin{array}{l}\text { SURFACE } \\
\qquad \quad \text { Parosteal } \\
\text { • Periosteal } \\
\text { High grade surface }\end{array}$ \\
\hline Cartilage-forming tumours & $\begin{array}{l}\text { Enchondroma } \\
\text { Osteochondroma } \\
\text { BPOP } \\
\text { Subungual exostosis } \\
\text { Chondroblastoma } \\
\text { Chondromyxoid fibroma }\end{array}$ & Mesenchymal chondrosarcoma \\
\hline Fibrous/Osteofibrous tumours & $\begin{array}{l}\text { Non-ossifying fibroma } \\
\text { Fibrous dysplasia } \\
\text { Osteofibrous dysplasia }\end{array}$ & \\
\hline Osteoclast-rich tumours & $\begin{array}{l}\text { Aneurysmal bone cyst } \\
\text { Giant cell tumour of bone }\end{array}$ & $\begin{array}{l}\text { Malignant giant cell tumour of } \\
\text { bone }\end{array}$ \\
\hline $\begin{array}{l}\text { 'Small round blue cell tumours' } \\
\text { (non-matrix producing) }\end{array}$ & & $\begin{array}{l}\text { Ewing Sarcoma } \\
\text { Lymphoma }\end{array}$ \\
\hline
\end{tabular}




\begin{tabular}{|l|l|l|}
\hline Miscellaneous Origin & Langerhans cell histiocytosis & $\begin{array}{l}\text { Adamantinoma } \\
\text { (transformation of osteofibrous } \\
\text { dysplasia) }\end{array}$ \\
& & $\begin{array}{l}\text { Chordoma (notochordal } \\
\text { differentiation) }\end{array}$ \\
\hline
\end{tabular}

Table 1. Classification of primary bone tumours 